\title{
Numerical Simulation OF WATER JeT QUALITY FOR DIFFERENT ORIFICE GEOMETRIES
}

\author{
GoikoetXeA, E.; Alberdi, A.; SuAREZ, A.; \\ ARLEO, F. \& LAMIKIZ, A.
}

Abstract: In plain water jet machining process $(P W J)$ an initial collision occurs between the jet and the wall of the orifice during the jet formation through a standard orifice, which disturbs the flow. The internal geometry has an important influence in the quality of the jet, and therefore in the cutting quality. This work presents a computational fluid dynamic (CFD) analysis with the aim of studying the effect of the internal geometry of the orifice on water jet characteristics. Different models were simulated modifying the upper part of a standard orifice. The results showed that the initial collision can be avoided and thus, disturbances on the external surface of the jet can be decreased modifying the internal orifice geometry, which leads to more stable cutting conditions. This analysis can serve in the future to optimize the internal geometry of the orifice and thus, to improve the characteristics of the water jet for each specific application, e.g., texturing or cutting.

Key words: turbulence, stability, CFD, orifice geometry, water jet
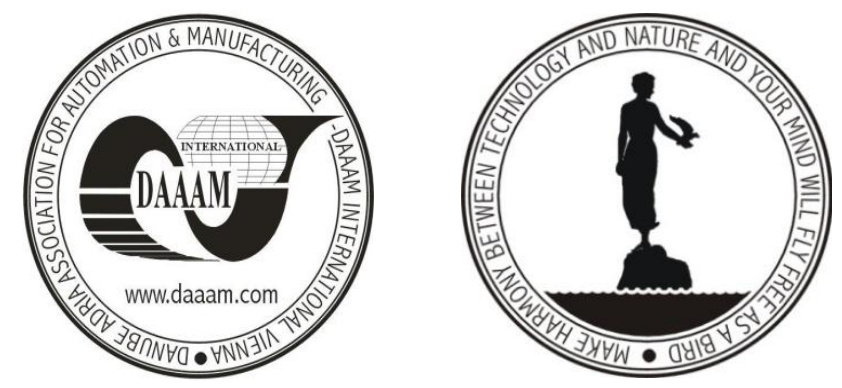

Authors' data: Dipl. Ing. Goikoetxea, E[stibalitz]*; Dipl. Ing. Alberdi, A[maia]*; Dipl. Ing. Suarez, A[lfredo]*; Dipl. Ing. Arleo, F[rancesco]**; Assoc. Prof. Lamikiz, A[itzol]*****Industrial Systems Unit, Manufacturing Engineering Department, Tecnalia, Paseo Mikeletegi 7, 20009, San Sebastian, Spain, ** Department of Mechanical Engineering, Politecnico di Milano, Via G. La Masa 1, 20156, Milano, Italy, **** Department of Mechanical Engineering, ETSI, University of the Basque Country, Alameda de Urquijo s/n, 48013, Bilbao, estibalitz.goikoetxea@tecnalia.com, amaia.alberdi@tecnalia.com, alfredo.suarez@tecnalia.com, francesco.arleo@mail.polimi.it, aitzol.lamikiz@ehu.es

This Publication has to be referred as: Goikoetxea, E[stibalitz]; Alberdi, A[maia]; Suarez, A[lfredo]; Arleo, F[rancesco] \& Lamikiz, A[itzol] (2011). Numerical Simulation of Water Jet Quality for Different Orifice Geometries, Chapter 41 in DAAAM International Scientific Book 2011, pp. 513-526, B. Katalinic (Ed.), Published by DAAAM International, ISBN 978-3-901509-84-1, ISSN 1726-9687, Vienna, Austria

DOI: $10.2507 /$ daaam.scibook.2011.41 


\section{Introduction}

Manufacturing requirements are increasing in terms of precision and productivity, for which novel processes are needed. Plain water jet cutting process (PWJ) is one of the most versatile and fastest processes. Its main advantage is that it can machine a wide range of materials without producing any thermal damage, thus, posterior surface treatments are avoided. Moreover, low forces involved during the process carry other advantages, such as the simplification of the clamping device system for the machined parts or the simplification of the design of the cutting head.

An essential step to improve the efficiency of the PWJ technology is to gain knowledge about the formation of the water jet and the effect of all parameters involved in that process, such as the internal geometry of the orifice (Fig. 2), which is the component responsible of transforming the hydrostatic energy of the water into a collimated jet with high kinetic energy. However, due to the high velocity and the small dimensions related to PWJ technology, it is difficult to make direct measurements and to get empirical information.

Hence, a useful tool for a better understanding of the jet formation is to make numerical simulations. In this sense, different works using CFD simulations can be found in the literature. E.g., the disturbances and the effect of droplets on the quality of the jet during the jet formation was studied (Arleo et al., 2010). The influence of the internal geometry of the orifice on the flow regime and on the discharge coefficient were analysed (Anantharamaiah et al., 2006a and 2006b), concluding that the ratio between the length and the diameter of the capillary $\left(L_{0} / d_{0}\right)$ has a strong influence on the flow regime. Also, a study was made (Basha et al, 2010) concluding that the internal geometry of the orifice housing affects to the velocity and the coherence of the jet and thus, to the cutting quality. They also studied the influence of the upper part of the orifice on the flow regime and the variation of the discharge coefficient during the initial instants of the formation of the jet (Basha et al., 2009).

From these studies it can be concluded that the internal geometry of the orifice has an important influence on the cutting efficiency of the jet, which depends on its coherence, its kinetic power, its continuity and the stability of its external surface, i.e., the part in contact with the surrounding air.

In order to find new solutions for improving the PWJ technology, the main goal of the presented work is to study alternative orifice geometries beyond the standard orifice studied in previous work made by Arleo et al. (2010). Hence, this work presents numerical simulations of the water jet flow through four new orifices with a modified upper part. This work allowed proposing new orifice geometries for different processes.

\section{Model Formulation}

The geometry and the initial mesh of the model were created using the Gambit 2.4 software and the model simulations were calculated using Fluent 6.3 software. 


\subsection{Goberning Equations}

The flow were considered as a turbulent, multiphase, incompressible and unsteady flow formed by air (primary phase) and water (secondary phase). The hypothesis used by some researchers (Cadavid-Giraldo, 2004; Tabernero et al. 2010) confirms that the flow from the exterior surface of the jet is laminar due to sudden reduction of section at orifice inlet. Besides, the hypothesis says that flow remains turbulent at the core of the jet and that the laminar layer becomes turbulent after travelling a certain distance (Fig. 1.Fehler! Verweisquelle konnte nicht gefunden werden.).

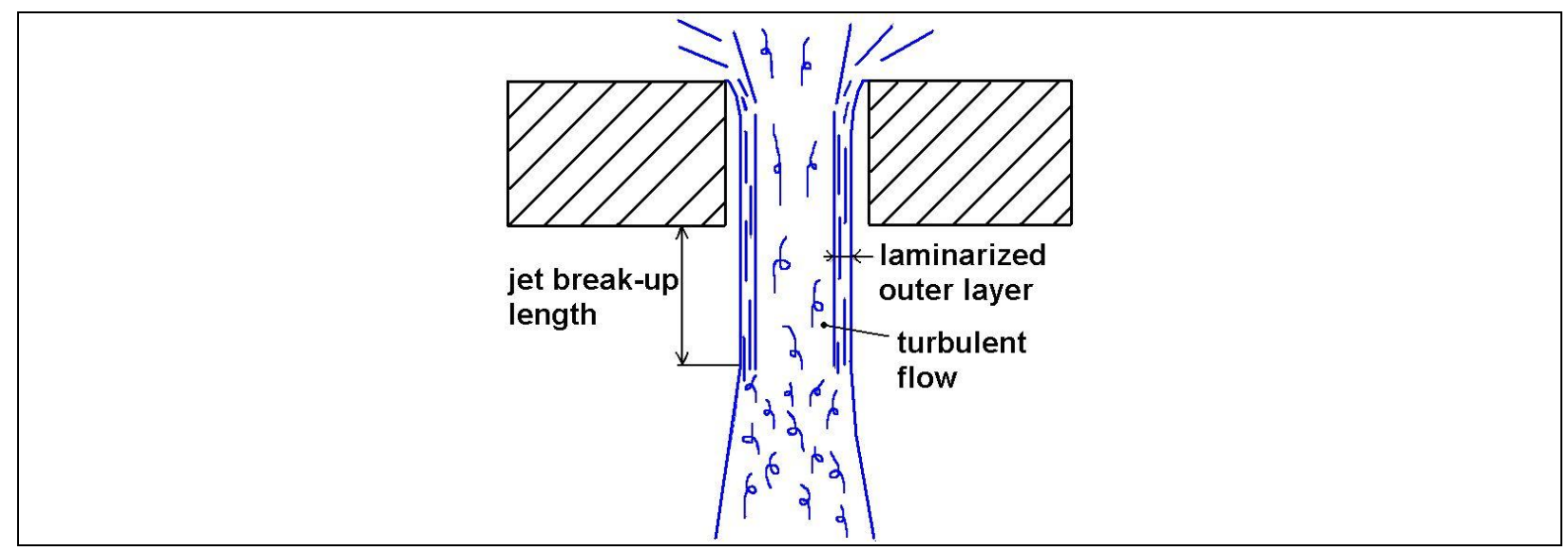

Fig. 1. Laminar regime flow at the outer layer of the jet

The system of equations is formed by: i) Reynolds Averaged Navier-Stokes Equations (RANS) equations, composed by the continuity equation for secondary phase (eq. 1) and momentum equations for velocity in $x$ and $y$ directions (eq. 2), and ii) turbulent viscosity model equations, composed by the turbulent kinetic energy $(k)$ equation (eq. 3 ) and the turbulent dissipation rate ( $\varepsilon$ ) equation (eq. 4).

$$
\begin{gathered}
\frac{\partial}{\partial x_{i}}\left(\alpha_{q} \bar{u}_{i}\right)=0 \\
\frac{\partial \bar{u}_{i}}{\partial t}+\frac{\partial}{\partial x_{j}}\left(\bar{u}_{i} \bar{u}_{j}\right)=-\frac{1}{\rho} \frac{\partial \bar{p}}{\partial x_{i}}+\frac{\partial}{\partial x_{j}}\left[v\left(\frac{\partial \bar{u}_{i}}{\partial x_{j}}+\frac{\partial \bar{u}_{j}}{\partial x_{i}}\right)+\tau_{i j}\right] \\
\frac{\partial k}{\partial t}+\bar{u}_{j} \frac{\partial k}{\partial x_{j}}=\tau_{i j} \frac{\partial \bar{u}_{i}}{\partial x_{j}}-\varepsilon+\frac{\partial}{\partial x_{j}}\left[\left(v+\frac{v_{t}}{\sigma_{k}}\right) \frac{\partial k}{\partial x_{j}}\right] \\
\frac{\partial \varepsilon}{\partial t}+\bar{u}_{j} \frac{\partial \varepsilon}{\partial x_{j}}=\frac{\partial}{\partial x_{j}}\left[\left(v+\frac{v_{t}}{\sigma_{\varepsilon}}\right) \frac{\partial \varepsilon}{\partial x_{j}}\right]+C_{1} S \varepsilon-C_{2} \frac{\varepsilon^{2}}{k+\sqrt{v \varepsilon}}
\end{gathered}
$$

Where $\alpha_{q}$ is the volume fraction of secondary phase, $\tau_{i j}$ are Reynolds stresses in $x_{i}$ and $x_{j}$ coordinate directions, $v$ is the molecular viscosity, $v_{\mathrm{t}}$ is the turbulent 
viscosity, $\sigma_{k}$ and $\sigma_{\varepsilon}$ are turbulent Prandtl numbers, $C_{1}$ and $C_{2}$ are coefficients and $S_{i j}$ are components of the Reynolds stress tensor.

The RANS equations used for solving the flow, calculate averaged characteristics of the turbulent flow, such as the pressure $\bar{p}$, the velocity in $x$ direction $\bar{u}$, the velocity in $y$ direction $\bar{v}, k$ and $\varepsilon$.

\subsection{Inlet data}

Following the previous work made by Arleo et al. (2010), same boundary conditions were introduced for the presented study. This makes possible to compare the results of the standard orifice with the proposed orifice geometries. These conditions consists on: (i) inlet pressure of $160 \mathrm{MPa}$ with turbulent intensity of $0.5 \%$ and hydraulic diameter of $3 \mathrm{~mm}$; (ii) outlet pressure of $101325 \mathrm{~Pa}$ with turbulent intensity of $4 \%$ and length scale of $1.225 \mathrm{~mm}$; (iii) non-slip condition and contact angle of $76^{\circ}$ at the wall and (iv) axis-symmetry at jet centreline. $K-\varepsilon$ realizable turbulent model, VOF multiphase model and PISO pressure-velocity coupling algorithm available in Fluent 6.3 were chosen.

\subsection{Standard Geometry}

The standard geometry showed in Fig. 2, which is used in industry for WJ process, has a cone-down configuration (with the capillary and the conical zone at the upper part), a sharp inlet edge and a ratio $L_{0} / d_{0}$ equal to 1 .

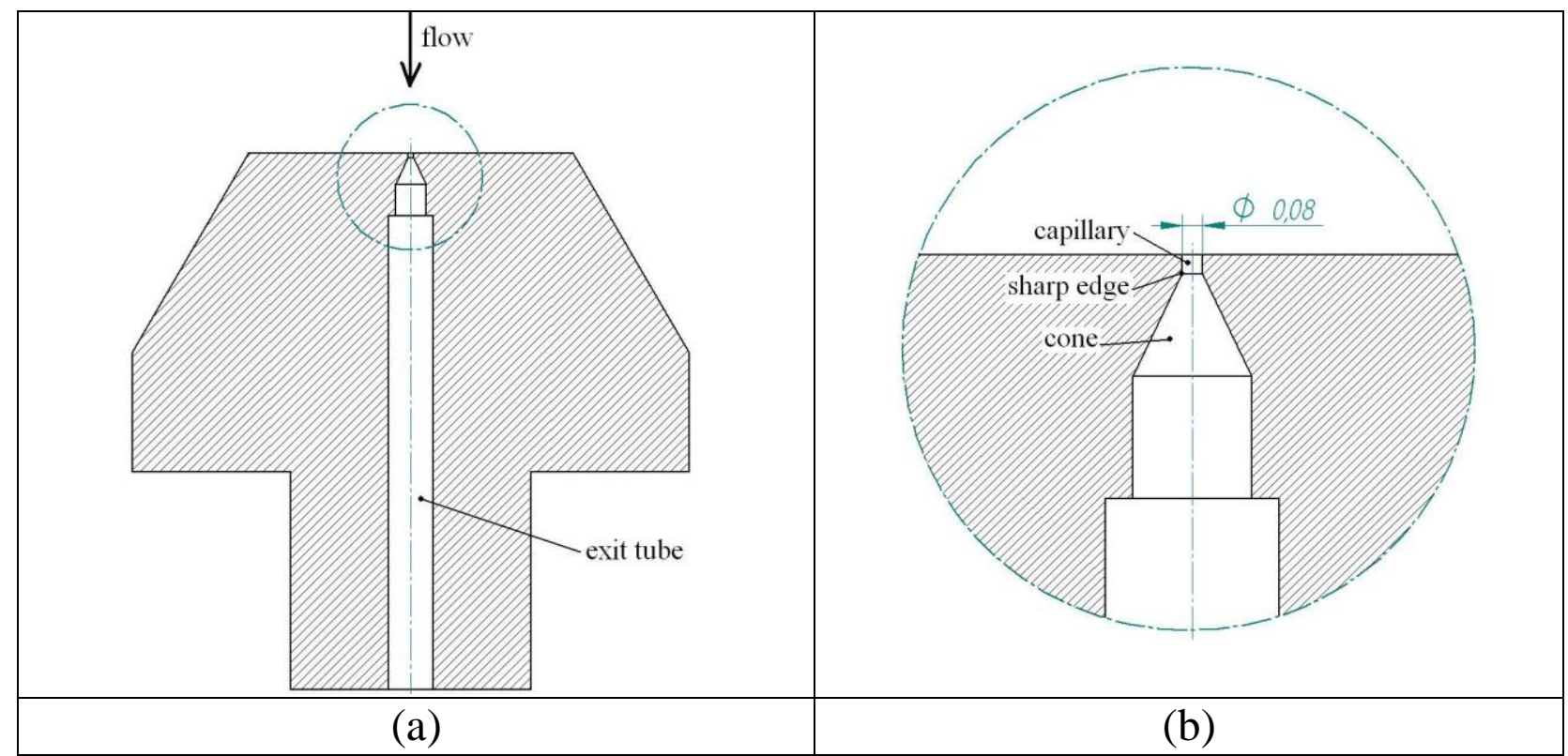

Fig. 2. (a) Standard orifice; (b) Upper part of the standard orifice

\subsection{Proposed Geometries}

The capillary is a critical zone where the generated chaotic air flow causes high jet friction losses. Different modifications of the upper part of the standard orifice were proposed with the aim of avoiding an initial collision and disintegration of the jet, and generating a smoother air flow, which leads to decrease energy losses and to 
improve the machining capacity of the jet. The proposed geometries are described below (Fig. 3):

- Conical geometry: the capillary is deleted to increase the space between the jet and the wall.

- Rounded geometry: the internal sharp edge between the capillary and the conical zone is rounded to generate a smoother transition of air upwards to the capillary. Different radiuses of curvature of internal edge $\left(r_{i n t}\right)$ were tested in a range between 0.1 and $0.3 \mathrm{~mm}$, and there were not found any difference between them. A $r_{\text {int }}$ of $0.3 \mathrm{~mm}$ was chosen.

- Short capillary geometry: the length of the capillary is reduced to L/2 to try reducing the maximum turbulence values placed near the capillary.

- Double conical geometry: the capillary is replaced with a progressive reduction inlet instead of the sudden reduction inlet to increase the space between the jet and the wall, and to maintain the strength of the orifice for the inlet pressure.

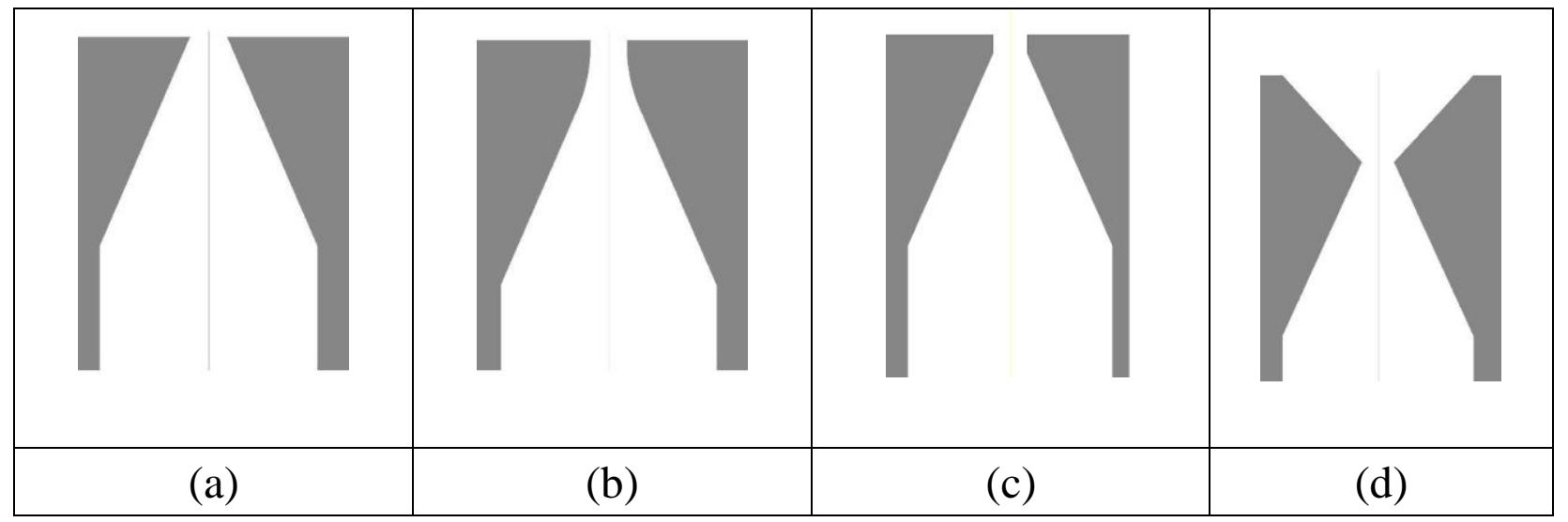

Fig. 3. Upper part of the orifice: (a) Conical geometry; (b) Rounded geometry; (c) Short capillary geometry; (d) Double conical geometry

The strength of the orifice for the inlet pressure is decreased when the capillary is reduced or even deleted, which entails a reduction in the life of the orifice. This is the case of the conical orifice, the short capillary orifice and the double conical orifice. The greatest difficulty regarding to the manufacturing of the orifices is to get the interior rounded edge between the capillary and the cone for the rounded orifice.

\section{Validation}

The simulation results of the proposed geometries were validated comparing them with theoretical data and the data from literature. A limitation of this work is that experimental validation of these models couldn't be achieved since the proposed orifices couldn't be manufactured. However, the experimental validation made for a standard orifice in previous work (Arleo et al., 2010) allowed to assume the model to be correct for the presented analysis, since the flow conditions and the orifice dimensions of the proposed geometries are similar to the standard one: orifice inlet diameter $d_{0}$ of $0.08 \mathrm{~mm}$, orifice length $H$ of $8.5 \mathrm{~mm}$, inlet pressure $P_{\text {inlet }}$ of $160 \mathrm{MPa}$, jet velocity $v$ of $567 \mathrm{~m} / \mathrm{s}$ and Reynolds number $R e$ of $4.5 \cdot 10^{4}$. This validation was done 
using a high speed camera and a pressure sensor, and an error lower than $0.15 \%$ was obtained.

\subsection{Discharge coefficient}

The discharge coefficient $C_{d}$ relates the real volume flow with theoretical volume flow. For its calculation the contraction coefficient $C_{C}$ obtained from the simulation and the velocity coefficient $C_{v}$ of 0.98 obtained from literature were used (eq. 5), this results in a discharge coefficient of 0.80 for the double conical geometry and of 0.62 for the rest of the geometries. These values are in agreement with the experimental results (Anantharamaiah et al., 2006b), which observes a discharge coefficient of 0.9 for cone-up nozzle, and a discharge coefficient of 0.62 for a sharp edge inlet.

$$
C_{d}=C_{c} \cdot C_{v}=C_{c} \cdot 0.98
$$

\subsection{Velocity}

Theoretical velocity at jet centreline at a distance of $0.08 \mathrm{~mm}$ from the orifice inlet calculated with the Bernoulli's theory is of $566.2 \mathrm{~m} / \mathrm{s}$. The values obtained in simulation were similar, a velocity of $567.7 \mathrm{~m} / \mathrm{s}$ and $568.3 \mathrm{~m} / \mathrm{s}$ were obtained for the conical and the double conical geometry, and for the rounded and the short capillary geometry respectively.

\subsection{Flow Regime}

Three possible flow regimes can be achieved in numerical simulations: i) a hydraulic-flipped regime, where a coherent jet surrounded by air is obtained; ii) a cavitating regime, where cavitation occurs inside the capillary; and iii) a single phase regime, where the flow fills the entire capillary.

Comparison between the flow regime achieved in the simulation and the flow regime predicted using a procedure for sudden reduction inlet orifices (Fluent 6.3 User's Guide, 2006) was done. However, this procedure is not appropriate to progressive reduction inlet, like double conical orifice presented here, so it was not applied in this case.

According to this procedure, the relation between the cavitation coefficient $K$, the critical value for cavitation $K_{\text {incep }}$ and the critical value for hydraulic-flip $K_{\text {crit }}$ predicts the flow regime. In all simulated and all predicted cases hydraulic-flipped regime was achieved, which supports that the model formulation is correct.

\subsection{Relation Between Turbulence and Velocity Losses}

The relation between the turbulence level and the velocity losses was observed. Due to high velocity field and high Reynolds values through the flow, main energy losses are generated due to the high turbulence level and the friction losses were considered null during the simulations. Hence, the turbulence level of the flow has a direct influence on jet velocity losses of the jet. 


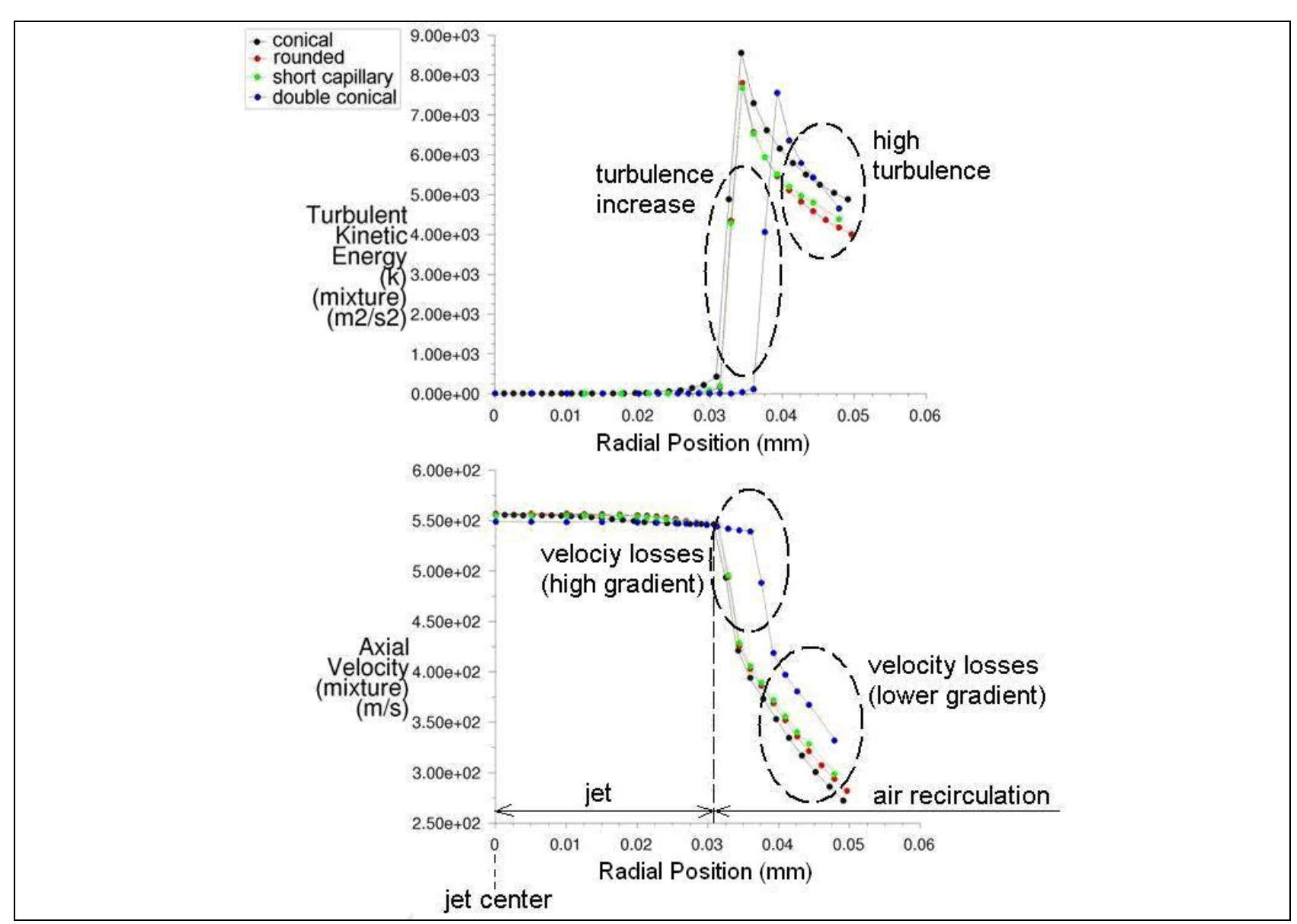

Fig. 4. Relation between turbulence and axial velocity losses

This is in accordance with the results obtained in the simulations, where zones with high turbulence values coincide with a decrease of the axial velocity of the jet. The analysis of the jet cross section at stand-off distance of $2 \mathrm{~mm}$ (Fig. 4), shows a high upslope of $k$ followed by a high turbulence level around the jet, due to the high velocity of surrounding air. This slope coincides with high axial velocity losses in external surface of the jet, which is in accordance with to theoretical assumption of the relation between turbulence level and the velocity losses and thus, supports the validation of the model.

\section{Results and Discussion}

All results, with the exception of the possible initial collision, correspond to stabilized flow, when the jet is already formed and the flow regime is permanent. Different orifice geometries did not show a significant difference regarding on the turbulence, the coherence and the cutting velocity profile. However, the differences are significant regarding the stability of the cutting velocity, the kinetic power and the strength of the orifice.

\subsection{Turbulent Kinetic Energy}

Turbulent kinetic energy parameter, $k$, is observed to analyze the turbulence level of the flow. Results show that the maximum $k$ values are concentrated in the air that is flowing inside the capillary zone. In standard geometry, which is the geometry 
that achieves the highest turbulence level, the maximum value is near the internal sharp edge between the capillary and the cone zone (Fig. 5a). In this point, high gradient of air velocity, high air velocity values and a chaotic air flow occurs (Fig. $5 b)$, due to the narrow space and to the internal sharp edge.

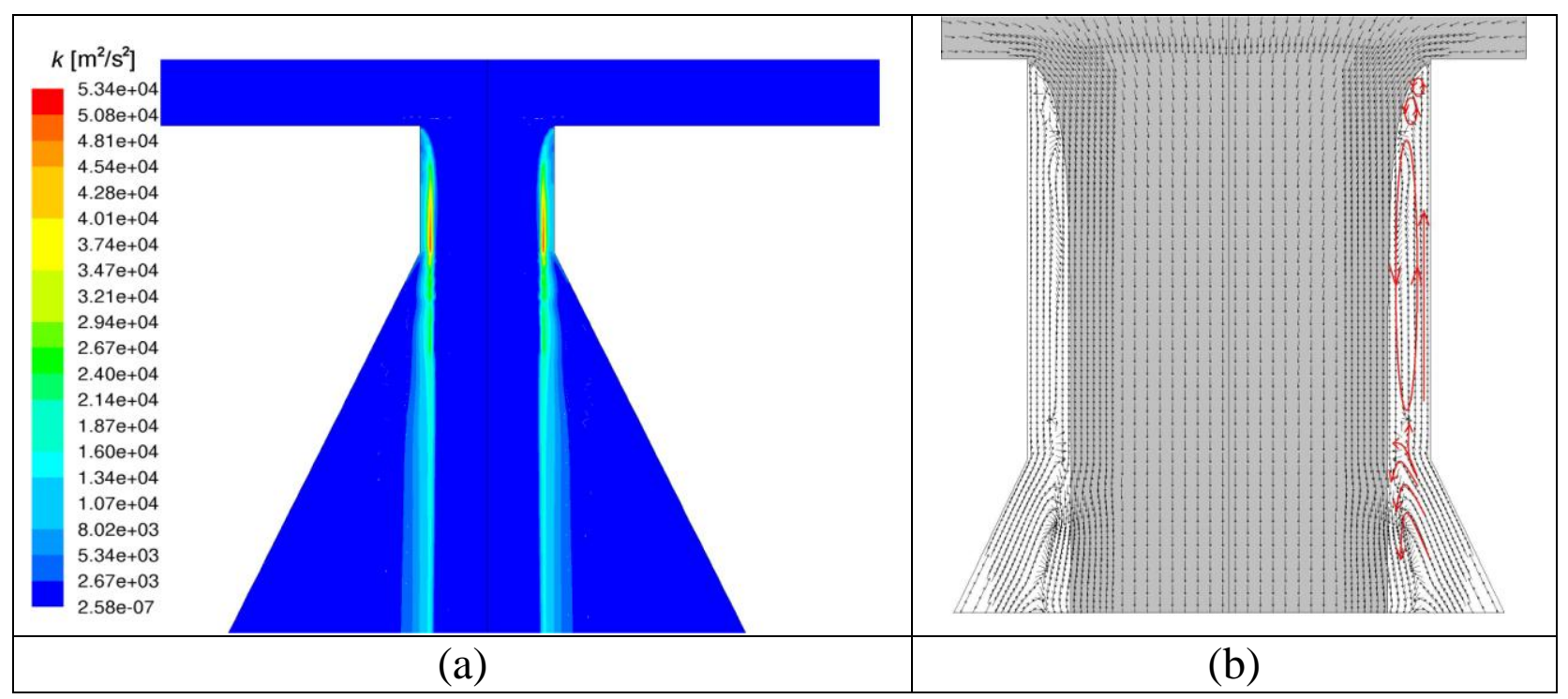

Fig. 5. Zone of maximum $k$ for standard geometry; (b) Air recirculation upper zone

The results showed that if the sharp edge is rounded or eliminated the maximum $k$ values are reduced (Tab. 1).

\begin{tabular}{|c|c|c|c|c|c|}
\hline & Standard & Conical & Rounded & Short capillary & Double conical \\
\hline $\boldsymbol{k}_{\max }\left[\mathbf{m}^{\mathbf{2}} / \mathbf{s}^{\mathbf{2}}\right]$ & $5.34 \cdot 10^{4}$ & $2.13 \cdot 10^{4}$ & $3.34 \cdot 10^{4}$ & $3.16 \cdot 10^{4}$ & $1.87 \cdot 10^{4}$ \\
\hline $\boldsymbol{k}_{\text {stand-off }}\left[\mathbf{m}^{\mathbf{2}} / \mathbf{s}^{\mathbf{2}}\right]$ & 811.335 & 2456.92 & 801.847 & 1044.21 & 383.644 \\
\hline
\end{tabular}

Tab. 1. Values of maximum $k$ and $k$ at stand off distance of $2 \mathrm{~mm}$

Regarding the entire domain, a layer of high turbulence level of air appears around the jet. The evolution of the value of $k$ along the jet exterior surface for different geometries is compared in Fig. 6. The effect of the variation of the internal geometry on the turbulence level at exit tube of the orifice or outside orifice is insignificant (Tab. 1).

Linking the turbulence values with the cutting velocity of the jet, it is concluded that the $k$ peak value of the inlet of the orifice does not affect on the cutting velocity of the jet at the exit of the orifice (Fig. 4). On the other hand, the high turbulence layer placed around the jet produces velocity losses on the jet. So it is more significant to focus analysing the stabilized turbulence level of the layer instead of the initial peak value.

Otherwise, the effect of the upper part of the internal geometry of the orifice produces minimal differences on this turbulence layer and thus, in the cutting velocity of the jet. Other zones of the internal geometry of the orifice can be modified in future works for a deeper study of the effect of the geometry. 


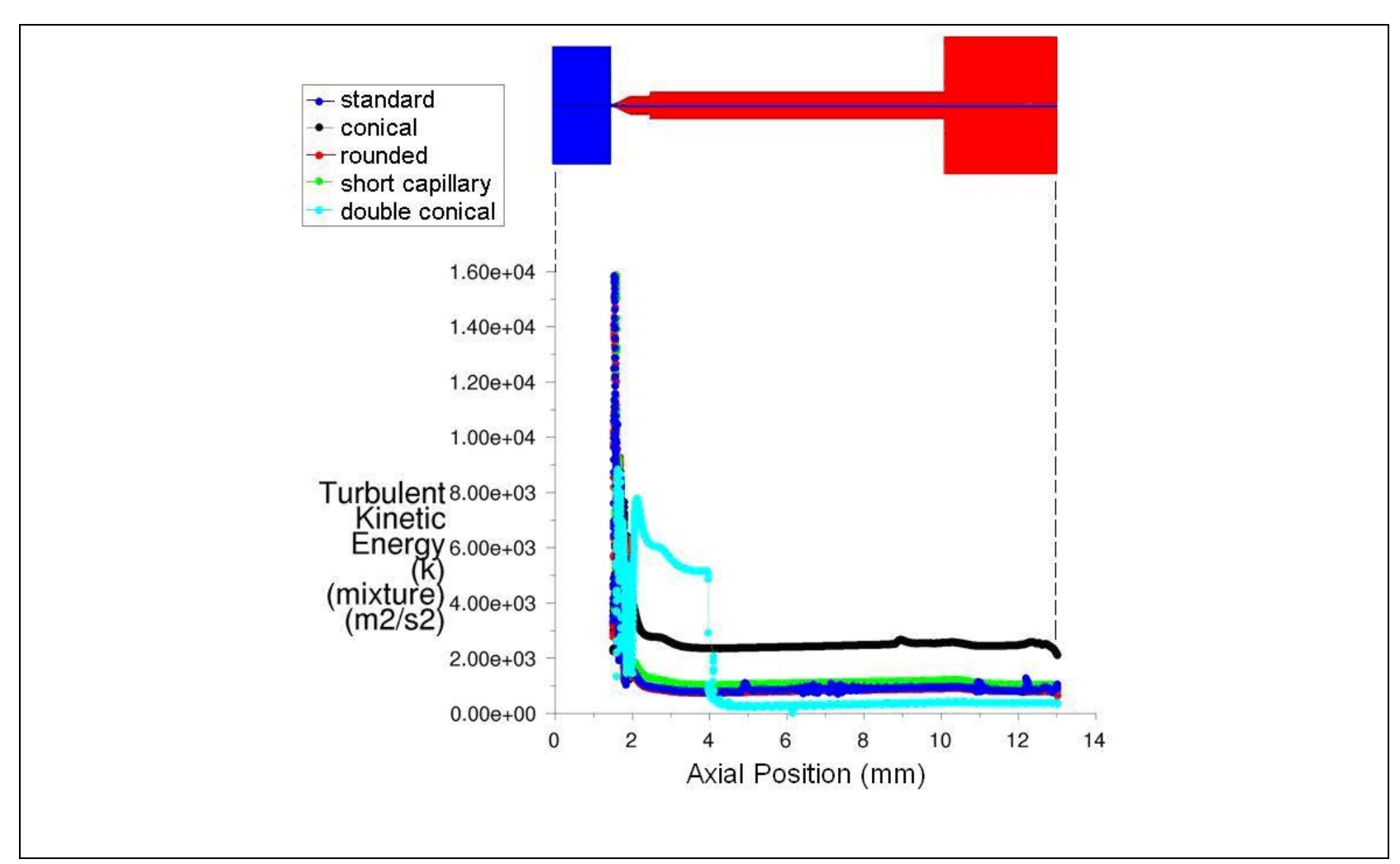

Fig. 6. Evolution of $k$ along external surface of the jet

\subsection{Coherence of the Jet}

A high coherence of the jet means that the jet is more stable and it losses less energy. Thus, it obtains greater cutting precision. Some of the factors which influence the jet coherence are the inlet pressure magnitude, the internal geometry of the orifice and the interaction between the outer surface of the jet and the surrounding air.

The parameter of the air volume fraction along the simulated domain is observed to analyze the coherence of the jet. In the case of a coherent jet, the visualization of this parameter shows a clear and straight separation line between the water phase and the air phase of the domain. Also, it shows the flow regime at the inlet of the orifice, e.g., a hydraulic-flip regime is obtained when the jet has a contraction at the inlet of the orifice.

The results showed that the flow reaches the hydraulic-flip condition for all the geometries. This condition is necessary to achieve a coherent jet. Besides the hydraulic-flip condition, flow simulations reached also a non slip condition between the jet and the air and a depression inside the orifice. Both conditions produce a recirculation of air around the jet inside the orifice. Air enters from the exit of the orifice and goes up close the wall until its upper part, where it turns to go down towards the exit generating a protection air layer around the jet and conserving the coherence of the jet.

The mean difference between standard geometry and the other geometries is the initial collision that occurs between the jet and the orifice when a standard orifice is used, which disintegrates the jet and causes droplets around the jet, damaging quality and coherence of the jet. In this case after this initial collision the flow 
separates again and reaches the hydraulic-flip regime. For the rest of orifice geometries the flow reaches directly this regime and the jet remains compact. This last situation is preferable to avoid possible disturbances due to an initial collision, so the proposed geometries improve the initial instants of the formation of the jet and thus, its quality.

\subsection{Cutting Velocity Profile in the Cross Section of the Jet}

The cutting velocity profile at jet cross section has an important influence in the cutting quality regarding to the taper angle. In practice, the taper angle is higher at the top surface of the work piece where the jet has a parabolic velocity profile. As the jet travels across the thickness of the work piece this profile becomes more uniform and the effective diameter of the jet is reduced. As a result, a more parallel kerf walls are produced.

Hence, it is preferable to get a uniform profile (Fig. 7) to reduce the taper angle and to improve the cutting quality. For surface treatments applications like texturing it is also better to get a uniform profile to erode a uniform depth at the work piece. The interaction between the jet and the air, which depends on internal geometry of the orifice, influences the velocity profile.

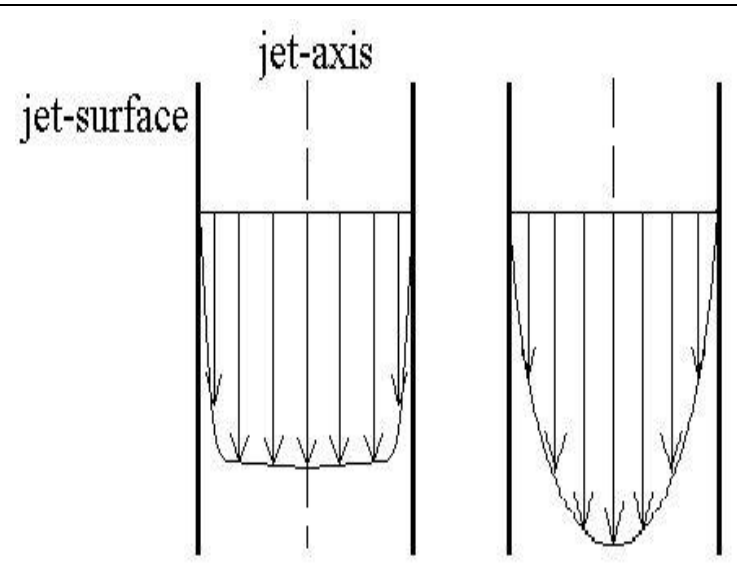

Fig. 7. Cutting velocity profile: uniform; parabolic

In Fig. 8 velocity profiles for different geometries at stand-off distance of $2 \mathrm{~mm}$ are compared. The least uniform velocity profile is obtained with the conical orifice, which will lead to a higher taper angle and a lower cutting quality of the work piece. On the other hand, the lowest mean velocity is obtained with double conical orifice (Tab. 2) and consequently, a lower kinetic power is achieved for the machining process. But the disadvantages of both orifices can be considered insignificant, because the influence of the geometry on the cutting velocity profile and on the mean cutting velocity is inappreciable.

The most significant difference regarding to velocity results is that the cutting velocity at external surface of the jet with the conical orifice is $3 \%$ lower $(15-20 \mathrm{~m} / \mathrm{s}$ lower) than in the other orifices (Tab. 2). In conclusion, variation of internal geometry of upper part of the orifice has not influence in cutting velocity of the jet. 


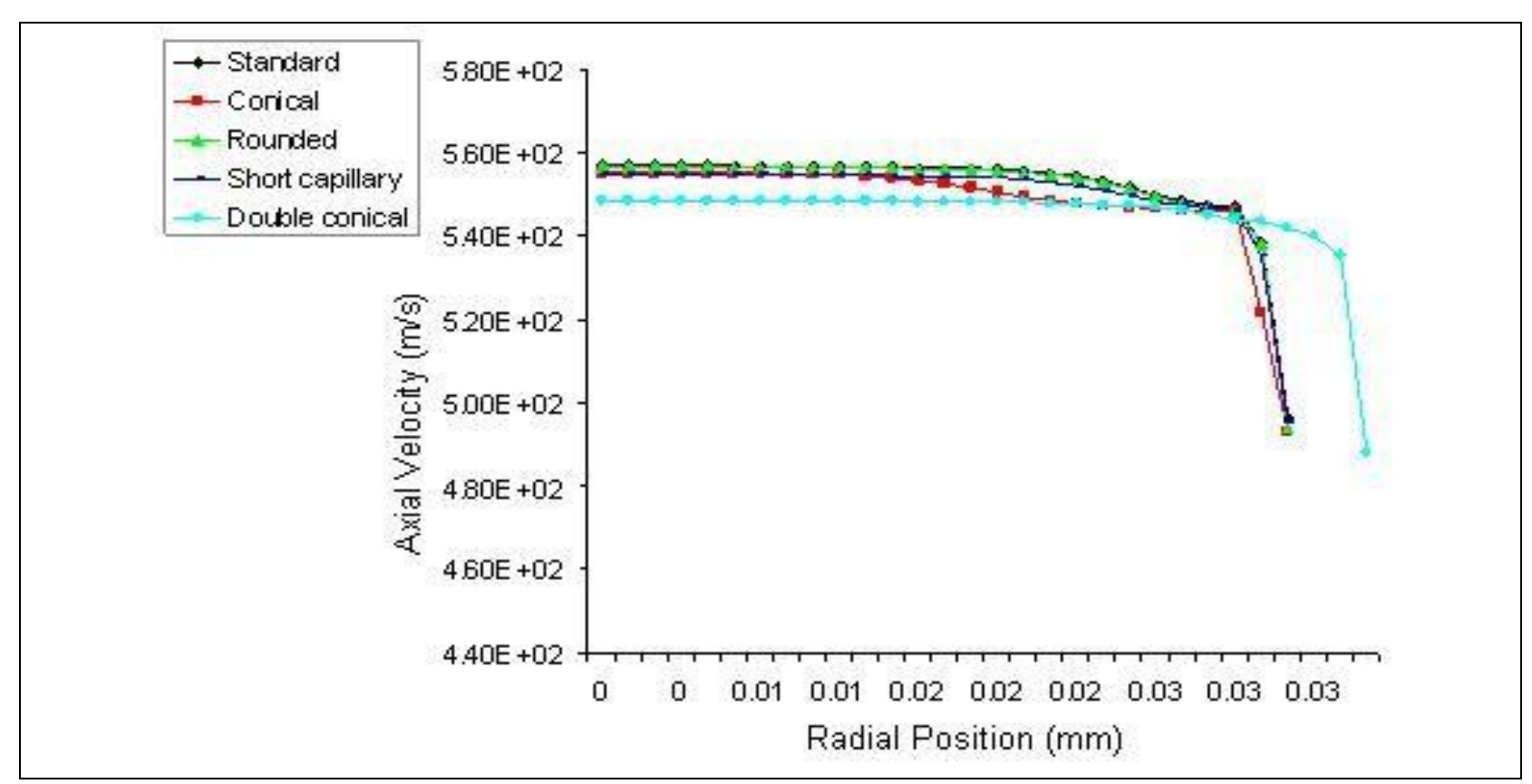

Fig. 8. Cutting velocity profile at stand-off distance of $2 \mathrm{~mm}$

\subsection{Stability of Cutting Velocity along the Jet}

To achieve a uniform and a repetitive cutting process, it is important to keep stable conditions along the external surface of the jet, since it is the part which is in contact with the work piece. Besides of internal instabilities, a factor which generates the disintegration of a jet is the instability of its external surface due to the resistance of the surrounding air.

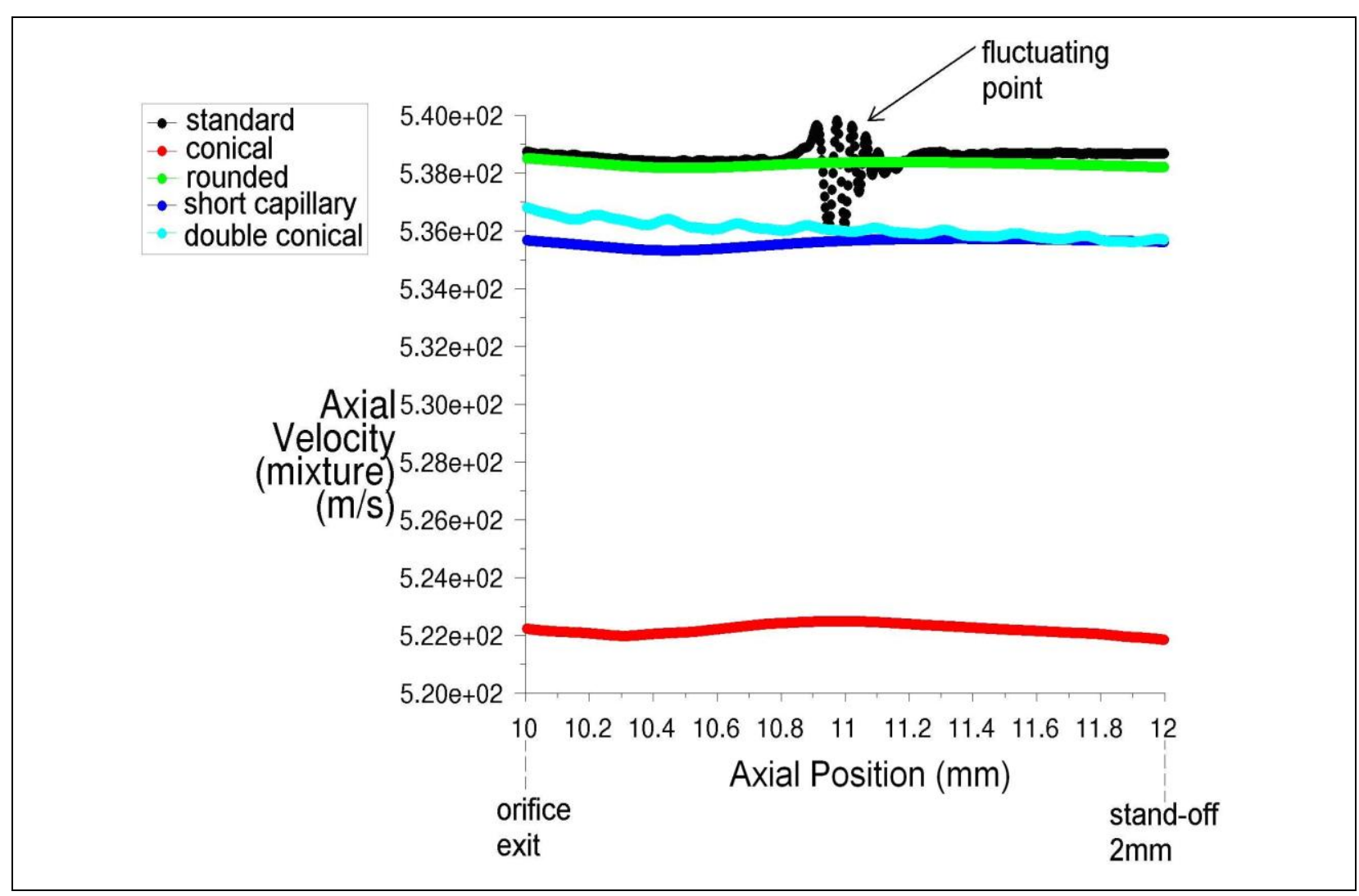

Fig. 9. Cutting velocity in the external surface of the jet 
Fig. 9 shows the curve of the cutting velocity along the external surface of the jet, outside the orifice, for different geometries. In the case of the standard geometry the cutting velocity shows fluctuations due to the initial collision of the jet, which damages the cutting quality. These fluctuations coincide with the presence of droplets or surface disturbances. The rest of orifices maintain a stable cutting velocity. Therefore, the proposed geometries improve the cutting quality of the jet, which is important to optimize the PWJ technology

\subsection{Kinetic Power of the Jet}

The kinetic power $\dot{E}$ depends both on the cutting velocity $v_{c}$ and on the water mass flow rate $\dot{m}$. So an increase of any these parameters produce a jet with a higher kinetic power, which leads to increase the cutting capacity of the jet and to decrease the machining time. In Tab. 2 the mean value of the kinetic power, calculated with the mean axial velocity of the cross section of the jet at a stand-off distance of $2 \mathrm{~mm}$, is compared for the simulated geometries, where standard orifice is considered as a reference.

\begin{tabular}{|c|c|c|c|c|c|}
\hline & Standard & Conical & Rounded & $\begin{array}{c}\text { Short } \\
\text { capillary }\end{array}$ & $\begin{array}{c}\text { Double } \\
\text { conical }\end{array}$ \\
\hline Exterior surface $v_{c}[\mathrm{~m} / \mathrm{s}]$ & 538.7 & 521.9 & 538.1 & 535.6 & 535.4 \\
\hline$\%$ variation & - & -3.1 & -0.1 & -0.6 & -0.6 \\
\hline Mean $v_{c}[\mathrm{~m} / \mathrm{s}]$ & 552 & 548.4 & 551.6 & 550.1 & 544.8 \\
\hline$\%$ variation & - & -0.7 & -0.1 & -0.3 & -1.3 \\
\hline$\dot{m}[\mathrm{~kg} / \mathrm{s}]$ & 0.0017 & 0.0017 & 0.0017 & 0.0017 & 0.0022 \\
\hline$\dot{E}[\mathrm{~W}]$ & 259.5 & 256.6 & 259.1 & 257.8 & 326.7 \\
\hline$\%$ variation & - & -1.1 & -0.2 & -0.7 & +25.9 \\
\hline Cross section $A\left[\mathrm{~mm}^{2}\right]$ & 0.0032 & 0.0032 & 0.0032 & 0.0032 & 0.0041 \\
\hline$\%$ variation & - & 0 & 0 & 0 & +30.6 \\
\hline
\end{tabular}

Tab. 2. Cutting velocity at stand-off distance of $2 \mathrm{~mm}$, kinetic power and cross section for each jet

The double conical orifice produces the jet with the highest mean kinetic power. Its progressive reduction inlet has an important influence in the flow. According to literature, it drives the water towards the narrow part of the orifice and produces less head losses during this initial reduction of the section (CadavidGiraldo, 2004).

Besides, this entry produces a jet with a higher diameter and a higher mass flow, while it maintains the similar values of cutting velocity of the jet comparing to the results obtained with the standard orifice. Thus, this orifice produces a jet with a higher machining capacity. The increase of the jet section leads to a wider contact surface between the top surface of the work piece and the jet, which allows eroding a wider surface per time and thus, reducing the machining time for surface treatment applications, as texturing. Rest of geometries produce a jet with similar diameter and kinetic power to standard geometry. In the other hand, for cutting applications and 
especially in micro-waterjet applications, a jet with a small diameter is better in order to reduce the kerf width and to reach higher precision.

\subsection{Summary of the Results for the Different Geometries} reference.

In Tab. 3 all the geometries are compared, taking the standard geometry as

\begin{tabular}{|c|c|c|}
\hline & Advantages & Disadvantages \\
\hline Standard & $\begin{array}{l}\text { - Orifice strength for the } \\
\text { inlet pressure }\end{array}$ & $\begin{array}{l}\text { - Initial collision } \\
\text { - Cutting velocity fluctuations along the jet }\end{array}$ \\
\hline Conical & $\begin{array}{l}\text { - No collision } \\
\text { - Stable cutting velocity }\end{array}$ & $\begin{array}{l}\text { - Lower orifice strength for inlet pressure } \\
\text { - Less uniform velocity profile } \\
\text { - Lower cutting velocity at jet external } \\
\text { surface }\end{array}$ \\
\hline Rounded & $\begin{array}{l}\text { - No collision } \\
\text { - Stable cutting velocity }\end{array}$ & $\begin{array}{l}\text { - Difficulty to manufacture the internal } \\
\text { rounded edge }\end{array}$ \\
\hline $\begin{array}{c}\text { Short } \\
\text { capillary }\end{array}$ & $\begin{array}{l}\text { - No collision } \\
\text { - Stable cutting velocity }\end{array}$ & - Lower orifice strength for inlet pressure \\
\hline $\begin{array}{l}\text { Double } \\
\text { conical }\end{array}$ & $\begin{array}{l}\text { - No collision } \\
\text { - Stable cutting velocity } \\
\text { - Further machining } \\
\text { capacity: surface } \\
\text { treatment, surface planing }\end{array}$ & $\begin{array}{l}\text { - Higher kerf width for cutting } \\
\text { applications, less precision: cutting, drilling } \\
\text { - Lower orifice strength for inlet pressure }\end{array}$ \\
\hline
\end{tabular}

Tab. 3. Summary of the results.

\section{Conclusions}

Depending on the WJ application, the optimal geometry of the orifice can be different due to the difference on the required jet characteristics for each application. The analysis made in the presented work can help to design the optimum orifice geometry for each application, for which the stability along the external surface of the jet and the size of the cross section should be taken into account.

For cutting or drilling applications, where narrow kerfs are required, the rounded geometry is found to be the best, since it improves the stability of the jet maintaining the rest of the jet characteristics the same as the standard orifice. Therefore, the rounded orifice allows achieving a better cutting quality than the quality obtained with the standard orifice.

On the other hand, for surface treatment applications as texturing, the double conical geometry is found to be the optimal geometry. Apart from improving the stability of external surface of the jet, it obtains also a jet with a higher machining capacity, because of its higher diameter. This jet allows eroding a wider surface of the work piece, reducing machining time for surface planing or texturing.

The most significant variation of the flow through the simulated geometries achieved in this work is the improvement of the stability of the jet. This is attained 
thanks to the elimination of the internal sharp edge, which leads to avoid the initial collision. Accordingly, the cutting velocity of external surface of the jet is more stable, without fluctuations of its value along the surface. This leads to improve the cutting quality of the work piece.

Future works should address the optimization of the orifice inner geometry for a specific application using CFD analysis, e.g, for surface treatment applications. The manufacturing of different orifice inner will be also a crucial step in order to prove the improvements of the presented analytical work.

\section{Acknowledgements}

This work has been carried out in the frame of the project Pro-Future of CIC marGUNE and supported thanks to the program Etortek 2010 of the Basque Government. We also wish to acknowledge to the "Fundación Centros Tecnológicos - Iñaki Goenaga" for supporting this work.

\section{References}

Anantharamaiah, N.; Vahedi Tafreshi, H. \& Pourdeyhimi, B. (2006a). Numerical simulation of the formation of constricted waterjets in hydroentangling nozzles: effects of nozzle geometry. Chemical Engineering Research and Design, Vol. 84, No. 3, pp. 231-238, ISSN

Anantharamaiah, N.; Vahedi Tafreshi, H. \& Pourdeyhimi, B. (2006b). A study on flow through hydroentangling nozzles and their degradation. Chemical Engineering Science, Vol. 61, No. 14, pp. 4582-4594, ISSN

Arleo, F.; Annoni, M.; Basha, A. T.; Etxeberria, I. \& Suarez, A. (2010). Numerical simulation of a pure water jet inside an orifice: jet stability and effect of droplets collisions. $20^{\text {th }}$ International Conference on Water Jetting, Trieb, F. H., pp. 301-316, ISBN, Austria, October 2010, BHR Group Limited, Graz

Basha, A. T.; Annoni, M. \& Monno, M. (2009). Numerical simulation of the formation and reattachment length of water for different orifice geometries. American WJTA Conference and Expo, Hashish, M., ISBN, Texas, August 2009, Houston

Basha, A. T.; Annoni, M.; Monno, M. \& Araneo, L. (2010). Effect of orifice housing geometry on pure water jet contouring performance. $20^{\text {th }}$ International conference on Water Jetting, Trieb, F. H., pp. 301-316, ISBN, Austria, October 2010, BHR Group Limited, Graz

Cadavid-Giraldo, R. (2004). Cutting with fluidjets of small diameter. Technische Universität Kaiserslautern, ISBN, Kaiserslautern

Fluent 6.3 User's Guide, 2006

Tabernero, I.; Lamikiz, A.; Ukar, E.; López de Lacalle, L.; Angulo, C. \& Urbikain, G. (2010). Numerical simulation and experimental validation of powder flux distribution in coaxial laser cladding. Journal of Materials Processing Technology, Vol. 210, No. 15, pp. 2125-2134, ISSN 